\title{
Soleus Activity in Post-Stroke SubJects: MOVEMENT SEQUENCE FROM STANDING TO SITTING
}

\author{
Augusta Silva ${ }^{1,2,3}$, Rita Pinheiro ${ }^{1}$, Andreia S. P. SousA ${ }^{1,2,4}$, JoÃo MANUEl R. S. \\ TAVARES $^{4}$, RuBim SANTOS ${ }^{2}$, FILIPA SOUSA ${ }^{3}$ \\ ${ }^{1}$ Àrea Cientifica de Fisioterapia, Escola Superior de Tecnologia da Saúde do Instituto Politécnico do Porto, Vila \\ Nova de Gaia, Portugal.smaugusta@gmail.com,arp@estsp.ipp.pt, asp@estsp.ipp.pt \\ ${ }^{2}$ Centro de Estudos de Movimento e Actividade Humana (CEMAH), Escola Superior de Tecnologia da Saúde do \\ Instituto Politécnico do Porto, Vila Nova de Gaia, Portugal.rss@estsp.ipp.pt \\ ${ }^{3}$ Laboratório de Biomecânica do Porto, Faculdade de Desporto, Universidade do Porto, Porto, Portugal. \\ filipas@fade.up.pt \\ ${ }^{4}$ Instituto de Engenharia Mecânica e Gestão Industrial, Departamento de Engenharia Mecânica, Faculdade \\ de Engenharia, Universidade do Porto.Porto,Portugal.tavares@fe.up.pt
}

\footnotetext{
* Corresponding author:

Àrea Científica de Fisioterapia, Escola Superior de Tecnologia da Saúde do Instituto Politécnico do Porto Rua Valente Perfeito, 322

4400-330, Vila Nova de Gaia, Portugal.

E-mail address: smaugusta@gmail.com (Augusta Silva).
}

Number of pages: 7

Number of tables: 1

Number of figures: 1

Declaration of interest: The authors report no conflicts of interest. 


\begin{abstract}
Introduction: The beginning of the movement sequence from standing to sitting requires the modulation of plantar flexors activity, including soleus muscle (SOL), to allow the forward translation of the tibia in relation to the foot, preserving its antigravity function. Purpose: To analyze the SOL activity during the initial phase of standing to sitting in stroke subjects. Methods: Two groups of ten subjects each participated in this study, one composed by healthy subjects and the other with subjects with history of stroke. Electromyographic activity (EMGa) of SOL was analyzed in ipsilateral (IPSI) and contralateral (CONTRA) limb to side lesion in stroke subjects, and in one limb in healthy subjects during the initial phase of standing to sitting. A force plate was used to identify the movement sequence phase. Results: The mean values of SOL EMGa were higher in healthy subjects than the ones obtained in IPSI and CONTRA limb in stroke subjects. Significance differences were only was observed between the IPSI and healthy limb ( $\mathrm{p}=0.035)$. Conclusion: When compared to the healthy subjects, stroke subjects showed a decreased SOL EMGa in IPSI limb, which suggests that therapeutic decisions must consider the need to promote a better postural control also in the IPSI limb.
\end{abstract}

KEY WORDS: Modulation of plantar flexors activity, ipsilateral and contralateral limb, postural control, electromyography.

\section{INTRODUCTION}

The adjustments in postural control to assume standing position in different sequences of movement, like walking and sitting, is the basis to perform multiple tasks that require the ability to generate postural tone. This postural tone should have an appropriate segmental distribution as well as a modulation of its variation according to the afferent input. There is evidence that the postural system, namely the ventro-medial system, is closely related to the level of soleus (SOL) muscle activity in many functional tasks (Horn, 2004). For example, during gait, specifically in the middle stance phase, the anterior displacement of the body involves the ability of the calf muscles to stretch with inherent regulation of their activity. Of these muscles, the SOL has a predominant focus of activity consistent with its inherent $\alpha$-motoneurons recruitment to dynamic stability of the ankle joint (Schneider et al., 2000).

In a previous study, we have found that in a group of five subjects with stroke history evaluated at mid-stance subphase of gait, four presented higher electromyographic activity (EMGa) of the SOL muscle in the contralateral (CONTRA) limb of the lesion of stroke and therefore predominantly impaired (Silva et al., 2011). However, sub-cortical injuries located at the internal capsule level, with dysfunction of the ventral-medial systems, may justify changes in the activity also in ipsilateral (IPSI) limb that may compromise the comparison between the CONTRA in relation to the IPSI as a control.

Several authors have reported that the changes observed in the gait pattern during the acute phase after stroke is due to a decrease in muscle strength, whereas biomechanical factors are the major cause of that changes in a post-acute phase (Lamontagne et al. 2002; Cruz, 2009). Proprioceptive sensory organs, i.e. spindles and Golgi tendon organs (GTO), play a key role in the proprioception of movement (Giullio et al., 2009).

During the mid-stance sub-phase, the modulation of afferent receptor type II and Ib allow an adequate tension regulation of the calf muscles according to the length variation without losing its postural stability 
ability (Giullio et al., 2009). It is known that only the active muscles promote afferent information from the neuromuscular spindle and GTO, among other mechanical receptors, to the central nervous system (CNS) (Raine et al., 2009).

In the beginning of the sequence from standing to sitting, the modulation of the calf muscles activity should allow the forward translation of the tibia in relation to the foot. The initial phase of this sequence depends on the modulation of Ib afferent fibers to allow the adjustable relation tension/length compatible with the maintenance of an antigravity postural control. Sitting requires the control of eccentric muscle contraction to allow the subject to control the descending of the body without a visual guidance (Roy et al., 2006).

Therefore, the main purpose of this work was to analyze the behavior of the SOL EMGa, during stand to sit, in CONTRA and IPSI of stroke subjects and compare it to healthy subjects.

\section{Methods}

\subsection{Participants}

This study included two groups: (1) the "healthy group" and (2) the "stroke subjects” group.

The "healthy subjects" group consisted of 10 individuals (six female and four male) with no history of neurological and/or orthopedic disorders. In this group were included subjects with age between 30 to 65 years that could be classified as sedentary persons according to the Centre for Disease Control for the American College of Sports Medicine (Thompson, 2001).

The "stroke subjects" group was formed by ten subjects (four female and six male) that suffered one isquemic stroke episode that resulted in hemiparesis. As inclusion criteria were also required that: the stroke episode involves an injury in the territory irrigated by the middle cerebral artery (MCA), namely the internal capsule (confirmed by brain computerized axial tomography), and an Fugl-Meyer Assessment of Sensorimotor Recovery After Stroke scale score inferior to 34 (Lamontagne et al., 2002).

The exclusion criteria assumed for both groups were: inability to perform sit to stand and stand to sit sequences independently; basic cognitive functions compromised (assessed by the Mini-Mental State Examination); history of other neurologic diseases (Parkinson disease, pontine and/or cerebellar lesions, sensory impairment); diabetes; thrombophlebitis; history of lower limb surgery and any orthopedic or rheumatoid conditions interfering with sit to stand and stand to sit sequences; and subjects that were under medication that could affect the motor performance.

Both groups show homogeneity in terms of age and height (Table 1).

This study was approved by the Ethics Committee of the Institutions involved. All subjects gave their informed consent according to the Declaration of Helsinki. 


\subsection{Instruments}

For the lower limb evaluation, the corresponding part of the adapted version to the Portuguese population of the FuglMeyer Assessment of Sensorimotor Recovery After Stroke was applied. The EMG signal was acquired and processed using the MP 150 Workstation model from Biopac Systems, Inc. (USA), and the Acqknowledge software (Biopac Systems, Inc. USA). A force plate, model FP4060-10 from Bertec Corporation (U.S.A), connected to a Bertec AM 6300 amplifier, with default gains, connected to a Biopac 16 bit analogical-digital converter and adopting a $1000 \mathrm{~Hz}$ sampling rate, was used to acquire the ground reaction force.

\subsection{Procedures}

\subsubsection{Electrode placement}

Immediately before the electrode placement, the skin was prepared to reduce the impedance to a level equal or inferior to $5 \mathrm{~K} \Omega$ (Camargos et al., 2009; Cheng et al., 2004; Correia \& Mil-Homens, 2004).

The electrode placement followed the recommendations of the European collection for Surface Electromyography (SENIAM) for the SOL muscle, being at the first $2 / 3$ of the line joining the medial condyle of the femur and the medial malleolus. The ground electrode was placed over the olecranon.

\subsubsection{Measurement}

After an explanation about the procedures, the subjects in barefoot and using shorts were instructed to maintain a standing position on the force platform with the arms parallel to the body (according to each subject aptitude) for 30 seconds. Subjects were instructed to initiate the movement of sitting after the verbal command "You may sit" (Cheng et al. 2004; Galli et al. 2008). A plinth was placed in height adjusted to $100 \%$ of the lower leg length (from the knee joint to the ground), as a reference for the subjects to assume the sitting position.
The individuals were instructed to perform the task at a normal speed without using the upper limbs or move their feet (Dubost et al. 2005), while maintaining a visual reference placed 2 meters away. One minute rest between each trial was adopted, and the necessary repetitions were performed in order to obtain three valid trials. On the stroke subjects group, both members were analyzed simultaneously, while on the healthy group each member was randomly selected. Electromyographic data were collected at $1000 \mathrm{~Hz}$.

\subsubsection{Data analysis}

The raw EMG signal and the force plate data was processed using the Acqknowledge software. The raw EMG signal was filtered by a band-pass filter of $20 \mathrm{~Hz}$ and $500 \mathrm{~Hz}$, and smoothed using a root mean square (RMS) algorithm (Lamontagne et al. 2000; Lamontagne et al. 2001; Billot et al. 2010). The signal from the force plate was also processed, using a low pass filter of $10 \mathrm{~Hz}$, and the force values were normalized according to the weight of each subject (Turns et al. 2007).

The moment onset, defined as time zero (T0), and the final phase were recognized using the anteroposterior component of groun reaction force $\left(\mathrm{F}_{\mathrm{AP}}\right)$. The beginning of sitting was defined as the time at which the signal exceeded the M+2SD (mean value plus twice the standard deviation value) value of the basal activity, during a time interval equal or superior to $50 \mathrm{~ms}$. The final phase was defined as the time at which the $\mathrm{F}_{\mathrm{AP}}$ changed its direction (Figure 1).

The onset of SOL relatively to T0 was identified by EMG, a rest interval was selected from -500 to $+500 \mathrm{~ms}$, being registered the $M$ and SD values. The beginning of the muscular activity was defined by the interval of time when the EMG signal exceeds the M+2SD value of the basal activity, during a time interval equal or superior to $50 \mathrm{~ms}$. The SOL EMGa 
magnitude was registered at the time interval studied. The obtained values were normalized to the muscle's maximum activity peak for the functional activity under study (Lamontagne et al. 2002).

All procedures were performed for each trial, having the arithmetic mean of the values obtained for each variable in three satisfactory trials been calculated. To ensure the reproducibility of the technique and reduce operator error, the data acquisition was performed always by the same investigator (Galli et al. 2008).

\section{STATISTICS}

Using descriptive statistics, the measures of central tendency (M) and dispersion (SD) for the magnitude of SOL EMG activity were calculated.

Considering the reduced size of both sample groups ( $\mathrm{n}=10)$, it was assumed that the variables did not follow a normal distribution. Thus, the Wilcoxon Signed Rank Test was applied to compare the magnitude of SOL EMG activity between both lower limbs in stroke subjects, and the Mann Whitney test to compare the stroke with healthy subjects, using the Statistical Package for the Social Sciences (SPSS IBM, USA). Differences with $\mathrm{p}<0.05$ were considered statistically significant.

\section{RESULTS}

In order to assess their variability, the "healthy subjects" and "stroke subjects" groups were characterized in relation to age, weight and height. As one can verify from Table 1, both groups were homogeneous in terms of age $(\mathrm{p}=0.724)$ and height ( $\mathrm{p}=0.343)$. The weight was higher in stroke subjects $(p=0.004)$. Moreover, the stroke subjects group shows a time evolution superior to two years.

The results concerned to the relative EMGa of the SOL in both limbs of stroke subjects and in healthy subjects are represented in Figures 2 and 3, respectively. The mean values of SOL EMGa were higher for the healthy subjects than the ones obtained in IPSI and CONTRA limb in stroke subjects. However, significance differences were only found between the IPSI and aleatory limb of healthy subjects $(p=0.035)$. When comparing IPSI and CONTRA SOL EMGa, the results demonstrated that the mean values were higher in CONTRA limb comparing to the IPSI limb in stroke subjects, but this was not statistical significant $(\mathrm{p}=0.333)$.

\section{DISCUSSION}

Previous studies show higher values of SOL EMGa during middle stance phase in the CONTRA limb to the side of the lesion (Silva et al. 2011). In spite of the non existence of statistical significantly differences, this finding leads to the need of verifying if during the execution of a task that demands a modulation of the SOL (according to length variation), like standing to sit, this tendency would be also maintained. This requirement is supported by Loram et al. (2007) when they state that the term passive can have absence of muscle activity but also the absence of activity modulation. Thus, the fact that there was EMG activity does not exclude the possibility of an alteration or even an absence of modulation which, according to the same authors, is a condition included in the passive postural control designations.

Studying the behavior of SOL muscle in the terminal stance phase and in the initial contact sub-phase would not be illustrative of the result of modulation of these afferents. In the terminal sub-phase the main requirement is related to an increased muscle power, and in the initial contact by an increase in the tibial anterior muscle activity (Gefen, 2001). 
The choice of the sequence from standing to sitting on its initial part, when occurs forward translation of the tibia in relation to the foot, associated to an anterior displacement of the center of pressure, is justified by the necessity of the modulation of afferent information by the GTO. Therefore, it can be hypothesized that, in this task, the antigravity stability, dependent from SOL modulation in relation to length and tension variation, is similar to the middle stance phase.

Our results do not show statistical significant differences between CONTRA and IPSI limb from SOL EMGa, in stroke subjects. It is important to note that the normalization procedure used in this study can be related to the non statistical significant results, since it can remove the true biological variation between measurements (Allison et al., 1993; Knutson et al., 1994). However, mean values were higher in CONTRA (23\%) in relation to IPSI (17\%) maintaining the tendency observed in our previous study (Silva et al., 2011). It should be noted that when in comparison to healthy subjects, the differences observed in stroke subjects between the limbs results from a decreased of SOL EMGa in IPSI limb and not from an increased of SOL EMGa in CONTRA limb. The statistical differences found between IPSI and healthy subjects may reflect less activity of SOL in IPSI limb, and can be explained with a possible lesion of corticalreticular system or a dysfunction of the reticular spinal system that is connected with postural control and has an ipsilateral disposition. So, these results can be justified by the involvement of the ipsilateral pathways that influence the postural control of muscles in IPSI limb, as the SOL. This results contradicted the idea that the main differences between stroke subjects and healthy subjects resides in CONTRA limb; however, the major part of the existent studies (Mazzaro et al., 2007;
Roy et al., 2006) do not assess the possible differences between IPSI and healthy subjects, which difficult the discussion of the results. Also, the studies that considered IPSI limb did not acquired the EMGa of SOL muscle; for a review, see (Olney and Richards, 1996).

Based on the fact that the stroke subjects showed a decreased of SOL EMGa in IPSI limb relatively to the healthy subjects, some clinical hypothesis can be formulated. To improve the functional movement, the therapeutic decisions must consider the need to promote a better postural control of the IPSI limb and do not be only focused on the CONTRA limb.

The understanding of the results obtained can be complemented with a discussion about the relationship between muscle activity and length variation. The largest source of proprioceptive information to the CNS results from the variation of muscle length ensuing from their muscle activity variations (Giullio et al. 2009). It is our believe that in standing to sitting the contribution of afferent fiber type $\mathrm{Ib}$ is crucial for the occurrence of modulation of muscle activity observed by the EMGa. This perception reinforces the need to assess the modulation of Ia and Ib afferent fibers through the register of the Hoffmann reflex during this task. The register of the SOL muscle length variation through the use of the ultrasonography may also allow this further study.

\section{REFERENCES}

Allison GT, Marshall RN \& Singer KP. 1993. EMG signal amplitude normalization technique in stretchshortening cycle movements. Journal of Electromyography and Kinesiology, 3(4): 236-244.

Billot M, Simoneau E, Hoecke J, Martin A. 2010. Coactivation at the ankle joint is not sufficient to estimate agonist e antagonist mechanical contribution. Muscle \& Nerve, 41(4): 511-518. 
Cheng PT, Chen CL, Wang CM, Hong WH. 2004.

Leg Muscle activation patterns of sit-to-stand movements in stroke patients. American Journal of Physical Medicine \& Rehabilitation, 83(1):10-16.

Cruz TH, Lewek MD, Dhaher Y. 2009. Biomechanical impairments and gait adaptations post-stroke: Multi-factorial associations. Journal of Biomechanics 42(11):1673-1677.

Dubost VD, Beauchet O, Manckoundia P, Herrmann F, Mourey F. 2005. Decreased trunk angular displacement during sitting down: an early feature of aging. Physical Therapy, 85(5):404-412.

Fletcher L, Cornall C, Armstrong S. 2009. Moving between sitting and standing. In: Raine S, Meadows L, Lynch-Ellerington M, editors. Bobath Concepttheory and clinical practice in neurological rehabilitation. United Kingdom: Wiley-Blackwell. pp. 83-116.

Galli M, Cimolin V, Crivellini M, Campanini I. 2008. Quantitative analysis of sit to stand movement: experimental set-up definition and application to healthy and hemiplegic adults. Gait \& Posture, 28 (1):80-85.

Gefen A. 2001. Simulations of the foot stability during gait characteristic of ankle dorsiflexor weakness in the elderly. Neural Systems and Rehabilitation Engineering, 9(4): 333-337.

Giulio ID, Maganaris CN, Baltzopoulos V, Loram I. 2009. The proprioceptive and agonist roles of gastrocnemius, soleus and tibialis anterior muscles in maintaining human upright posture. Journal of Physiology, 587(10): 2399-2416.

Horn ER. 2004. Critical periods in vestibular development or adaptation of gravity sensory systems to altered gravitational conditions? Archives Italiennes de Biologie, 142(3):155-174.

Knutson LM, Soderberg GL, Ballantyne BT \& Clarke WR. 1994. A study of various normalization procedures for within day electromyographic data. Journal of Electromyography and Kinesiology, 4(1):47-59.
Lamontagne JA, Richards C, Malouin F. 2000. Coactivation during gait as an adaptive behaviour after stroke. Journal of Electromiography and Kinesiology, 10(6):407-415.

Lamontagne JA, Malouin F, Richards CL, Dumas F. 2001. Mechanisms of disturbed motor control in ankle weakness during gait after stroke. Gait \& Posture 15(3):244-255.

Loram ID, Constantinos N, Lakie M. 2007. The passive, human calf muscles in relation of standing: the non-linear decrease from short range to long range stiffness. Journal Physiological Society, 584(2): 661-675.

Mazzaro N, Nielsen JF, Grey M, Sinkjaer T. 2007, Decreased Contribution From Afferent Feedback to the Soleus Muscle During Walking in Patients with Spastic Stroke. Journal of Stroke and Cerebrovascular Diseases, 16 (4):135-144.

Olney SJ, Richards C. 1996. Hemiparetic gait following stroke. Part I: Characteristics. Gait \& Posture, 4(2):136-148.

Raine S, Meadows L, Lynch-Ellerington M. 2009. Bobath Concept, Theory and Clinical Practice in Neurological Rehabilitation. United Kingdom: Wiley-Blackwell.

Ricamato A, Hidler J. 2005. Quantification of the dynamic properties of EMG patterns during gait. Journal of Electromyography and Kinesiology, 15(4): 384-392.

Roy G, Nadeau S, Grave, D, Malouin F, McFadyen BJ, Piotte F. 2006. The effect of foot position and chair height on the asymmetry of vertical forces during sit-to-stand and stand-to-sit tasks in individuals with hemiparesis. Clinical Biomechanics 21(6):585-593.

Schneider C, Lavoie BA, Capaday C. 2000. On the origin of the soleus H-Reflex modulation pattern during human walking and its task-dependent differences. Journal of Neurophysiology 83(5):28812890.

Silva A, Santos R, Sousa F. 2011. Actividade do solear nos dois membros inferiores em sujeitos com 
AVE - sub-fase média de apoio da marcha. In L. Roseiro \& A. Neto (Eds.), $4^{\circ}$ Congresso Nacional de Biomecânica. Coimbra, Portugal, ISBN: 978-98997161-0-0 Sociedade Portuguesa de Biomecânica, pp. 627-632, 2011.

Turns L, Neptune R, Kautz S. 2007. Relationships between muscle activity and anteroposterior ground reaction forces in hemiparetic walking. Archives of Physical Medicine Rehabilitation, 88(9): 1127-1135. 


\section{Figure Captions:}

Figure 1: Subphase of standing to sitting sequence analysed.

Figure 2: Mean values of SOL relative EMGa (\%) in an aleatory limb of healthy subjects and in the CONTRA and IPSI limb to the lesion of stroke subjects.

Figure 3: Raw signals obtained from one healthy subject and one stroke subject. 
FIGURES

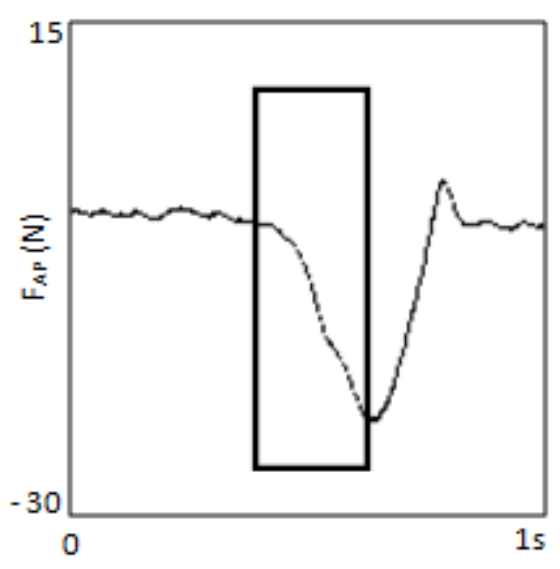

Figure 1

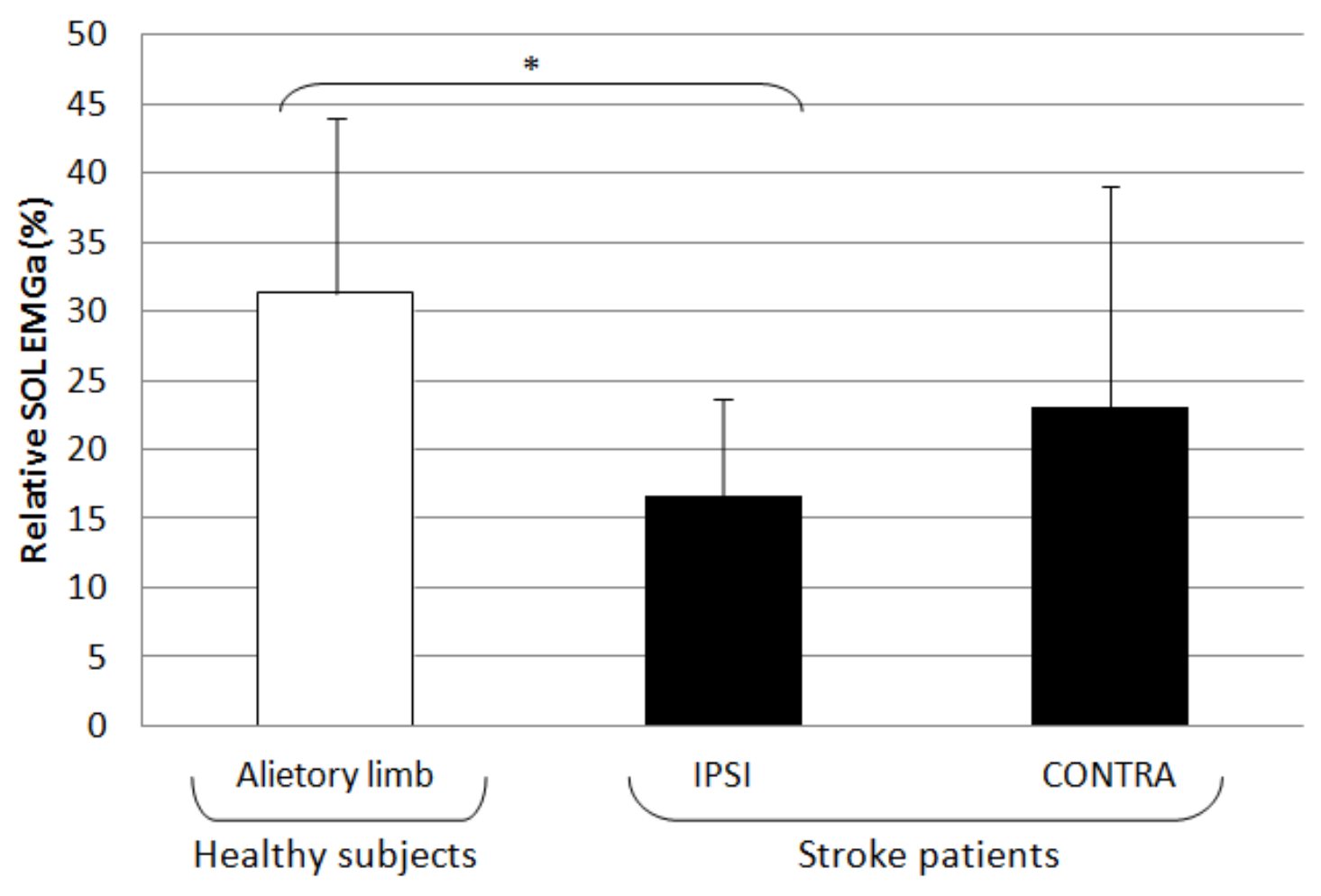

Figure 2 
Healthy subject

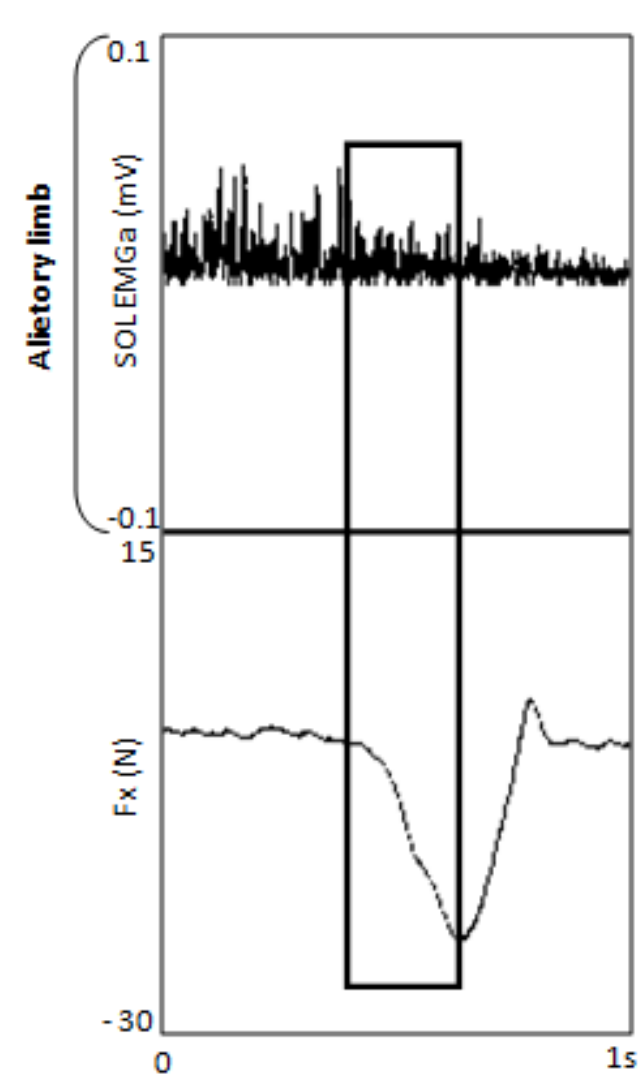

Stroke patient

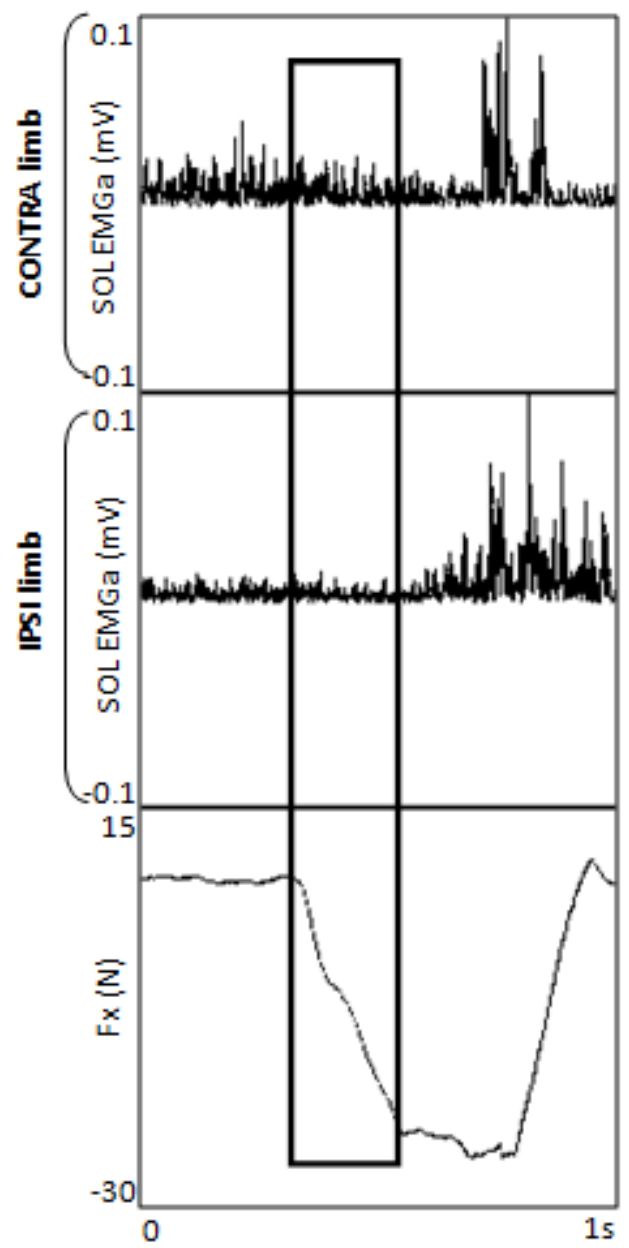

Figure 3 


\section{TABLES}

Table 1: Mean (M) and standard deviation (SD) values of age, height and weight of the healthy and stroke groups

\begin{tabular}{lcccc}
\hline \multicolumn{1}{c}{ Variable } & \multicolumn{2}{c}{ Healthy subjects } & \multicolumn{2}{c}{ Stroke subjects } \\
& $\mathrm{M}$ & $\mathrm{SD}$ & $\mathrm{M}$ & $\mathrm{SD}$ \\
\hline Age (years) & 50.4 & 10.8 & 49.3 & 8.53 \\
Height (cm) & 167.8 & 9.32 & 164.2 & 9.68 \\
Weight (Kg) & 67.6 & 6.66 & 80.9 & 8.98 \\
\hline
\end{tabular}

Table 2: Side lesion and time evolution of the stroke subjects.

\begin{tabular}{lc}
\hline \multicolumn{1}{c}{ Variable } & Stroke subjects \\
\hline $\begin{array}{l}\text { Clinical evolution } \\
\text { (months) }\end{array}$ & $28.6( \pm 14.91)$ \\
Side lesion & Right: $\mathrm{n}=3 \quad$ Left: $\mathrm{n}=7$ \\
\hline
\end{tabular}

